\title{
La Tarjeta Blanca de la Fundación Realmadrid. Una propuesta educativa a través de la competición.
}

\section{The white card fundación Realmadrid. An educative proposal through basketball competition}

\author{
Gema Ortega, Jorge Franco, Javier Giménez y Pedro Jiménez
}

PALABRAS CLAVE: Baloncesto, valores educativos, competición. RESUMEN: La competición en etapas de iniciación supone un excelente medio de aprendizaje para los jugadores/as, y una oportunidad magnífica de reforzar los conocimientos y actitudes transmitidas, para el profesorado. Por todo ello, desde las Escuelas de Baloncesto de la Fundación Realmadrid, y más concretamente desde el proyecto "Por una Educación REAL: Valores y Deporte" (Fundación Realmadrid, 2012), hemos centrado nuestros esfuerzos en elaborar una herramienta que nos permitiera aprovechar el potencial de la competición a nivel educativo. A través de nuestro modelo competitivo, que está basado en la demostración de buenas conductas y la aparición de valores positivos, decidimos implantar el programa Tarjeta Blanca. Este programa, que comenzó a desarrollarse en la temporada 2010-2011, consiste en una forma de reconocimiento a todos aquellos jugadores/as que demuestren actitudes y conductas positivas durante la propia competición. Nuestro objetivo es fomentar la aparición de esas conductas deseables, dando a conocer al alumnado cuáles de sus actos son dignos o merecedores de ser valorados positivamente. A su vez, creemos que con esta propuesta nuestros entrenadores/as también salen reforzados y esperamos que motivados, ya que estarán incentivados a observar y descubrir las conductas que pueden ser susceptibles de ser premiadas. Como dato más relevante deseamos mencionar que en la última edición del Torneo, se han premiado 308 conductas destacadas a nivel educativo sobre un total de 358 participantes, lo que demuestra la presencia y la relevancia del programa en nuestra competición.

KEY WORDS: Basketball, educative values, competition.

ABSTRACT: The competition in initiation to sport is an excellent learning environment for players, and a great opportunity for teachers to enhance knowledge and attitudes transmitted. Therefore, from Fundación Realmadrid Basketball Schools, specifically from the project "For a REAL Education: Values and Sport" (Realmadrid Foundation, 2012), we have focused our efforts on developing a tool that could exploit the potential competition on an educative way. Through our competition model, which is based on the demonstration of good behaviors and the appearance of positive values, we decided to implement the White Card program. This program, which began to develop in the 20102011 season, is a form of recognition to all players which demonstrate positive attitudes and behaviors during the competition. Our goal is to foster the emergence of these desirable behaviors, revealing the students which of their actions are worthy or deserving to be valued positively. In turn, we believe that with this proposal our teachers also are strengthened and hopefully motivated because they will be encouraged to observe and discover the behaviors that may be capable of being awarded. As most relevant data we want to mention that in our Tournament latest edition have been awarded a total of 308 behaviors leading to educational level over a total of 358 participants, demonstrating the presence and relevance of our competition program.

\section{Introducción}

La Fundación Realmadrid siempre ha pretendido utilizar el deporte como medio educativo y de desarrollo integral de la personalidad. La finalidad es claramente educacional y formativa, siguiendo una tendencia en la que el disfrute y diversión de los participantes es la clave en la búsqueda de los objetivos. Para ello, siempre hemos intentado que nuestra seña de identidad sea la transmisión de valores a través del deporte, y apoyándonos en esta idea, hemos desarrollado el proyecto "Por una Educación REAL: Valores y Deporte", en el que venimos trabajando desde la temporada 2008-2009

Dirección para correspondencia: Dña. Gema Ortega. Fundación Realmadrid. C.C. La Esquina del Bernabéu. C/ Padre Damián, 28036 Madrid.

E-mail: gortega@realmadrid.es

Este trabajo, enmarcado en el proyecto "Por una educación REAL: Valores y Deporte", ha sido financiado por la Fundación Realmadrid (2008-09/2011-12). con la intención de facilitar la aparición de valores educativos dentro de nuestras escuelas deportivas (Ortega, Sáenz-López, Durán, Giménez, Jiménez y Jiménez, 2011).

Dentro de esta filosofía, es importante para nosotros insistir en la idea de que los entrenadores/as son esa pieza imprescindible, sin la cual nuestros objetivos no se podrían cumplir (Ortega, 2007). La práctica del deporte, tanto en los entrenamientos como en la competición, se realiza en un contexto social que ofrece múltiples posibilidades de progreso personal y de relación con los demás participantes. Durán (2006) plantea que la práctica deportiva es un medio extraordinario para cultivar los valores en los niños/as y jóvenes, debido a lo atrayente y divertido que resulta ser su práctica. Destaca el carácter vivencial y las numerosas relaciones interpersonales del deporte que permite desarrollar las dimensiones reflexiva, conductual y afectiva, necesarias en una buena educación en valores. 
Como nuestro modelo deportivo busca una práctica del baloncesto diferente, la competición tiene que modificarse y adaptarse a las nuevas finalidades. Por ello, la competición de baloncesto en nuestras Escuelas Deportivas no tiene una filosofía tradicional (Jiménez y Ortega, 2010). Las finalidades de nuestra competición son claras: divertirse, relacionarse, educarse y aprender. Esto queda demostrado con algunas características propias del modelo de competición de las Escuelas de Baloncesto Fundación Realmadrid, como por ejemplo, el hecho de que los jugadores/as participen activamente en el arbitraje de los partidos, o que todos los jugadores/as jueguen el mismo tiempo durante los encuentros. El profesorado, el alumnado y la familia entienden que la puesta en práctica de nuestro modelo de competición es acorde a la filosofía que la Fundación Realmadrid intenta trasmitir. La competición se convierte en un estupendo medio de aprendizaje donde las reglas van a ser diferentes y se van a adaptar a cada edad, se va a buscar la máxima participación de los jugadores/as evitando la selección por nivel, y donde ni la clasificación ni el resultado son tan importantes.

Para ello, y en el contexto de este modelo de competición propio de nuestras Escuelas de Baloncesto, desarrollamos el programa Tarjeta Blanca (Ortega, Sáenz-López, Durán, Giménez, Jiménez y Jiménez, 2011), con el objetivo principal de fomentar conductas éticamente correctas entre el alumnado. El funcionamiento de este programa es claro y operativo, ya que los entrenadores tienen la posibilidad de entregar la Tarjeta a cualquier jugador/a participante en el Torneo en reconocimiento a ciertas conductas puntuales, o por su trayectoria general de deportividad. En este sentido, son los propios jugadores/as quienes dejan constancia por escrito de la conducta realizada en los paneles dedicados a ello y ubicados en la instalación deportiva donde se realiza la competición.

Tras una experiencia inicial en la cual los entrenadores/as eran los responsables de premiar directamente, la evolución del programa durante la última temporada (2011-2012) nos ha llevado a variar las pautas de actuación. En la parte final de cada partido, en una reflexión conjunta entre los dos equipos y el profesor/a, al finalizar el partido, pueden ser los propios jugadores/as quienes valoren los comportamientos y actitudes, y decidan cuáles de ellas son merecedoras de la Tarjeta Blanca. En base a esta última apreciación, establecemos un objetivo secundario, y no es otro que reforzar en nuestro profesorado su ya evidente sensibilidad educativa, transmitiéndola a su vez al alumnado.

\section{Método}

La metodología que se ha utilizado en nuestra investigación se engloba dentro del marco de la Investigación-Acción (Carr y Kemmis, 1988, Elliot, 1993), y más específicamente en la Investigación-Acción Participativa, en cuanto a que ha estado enfocada hacia el fomento de valores sociales y personales a través del deporte de un colectivo de jóvenes y entrenadores/ as de la Fundación Realmadrid. El programa se ha desarrollado durante dos temporadas, siendo partícipes del mismo un total de 19 entrenadores/as entre ambas. Es una técnica de carácter cualitativo que adopta una metodología de investigación abierta, democrática, centrada en los problemas prácticos de la educación y orientada a la mejora en la práctica de la misma, con vistas a mejorar los resultados y facilitar el progreso de las personas con las que se trabaja. Los instrumentos de investigación utilizados fueron la discusión de grupo, el cuestionario de satisfacción completado por parte de las familias, y las tablas de registros que recogen las conductas premiadas con la Tarjeta Blanca (tablón de competición y acta de partido).

Además de los datos obtenidos desde la implantación del proyecto, con el fin de analizar y evaluar con nuestros entrenadores/as el programa, desarrollamos un grupo de discusión con la participación de todos ellos. La intención fue reflexionar de manera conjunta sobre la experiencia que les estaba suponiendo la implementación del programa Tarjeta Blanca. Para nosotros, esta herramienta evaluativa es enormemente valiosa, ya que los entrenadores/as son la parte fundamental y representan la conexión directa con el alumnado, y nos pueden hacer llegar por tanto las sensaciones que se perciben en torno al proyecto. Durante la sesión, los entrenadores/as expusieron que consideraban la Tarjeta Blanca una iniciativa magnífica que refleja perfectamente la filosofía de la Fundación Realmadrid.

\section{Resultados}

El programa Tarjeta Blanca se incorporó al Torneo de las Escuelas de Baloncesto de la Fundación Realmadrid durante la celebración del mismo en la temporada 2010-2011. Durante esta primera temporada, se disputaron 13 jornadas de Torneo, los domingos desde febrero a junio, y se premiaron un total 75 conductas positivas, participando un total de 336 jugadores/a, pertenecientes a las categorías benjamín, alevín, infantil y cadete (niños/as entre 8 y 16 años por tanto). Una parte muy importante dentro del funcionamiento de nuestras escuelas es la opinión de las familias. Es por ello que como todos los años, al finalizar dicha temporada, las familias pudieron exponer sus opiniones y valoraciones dentro de la encuesta de satisfacción. En una tendencia general en la cual el trabajo en las Escuelas de Baloncesto está muy reconocido (84\% de los encuestados otorgan una buena-muy buena valoración global), el programa Tarjeta Blanca también fue positivamente valorado. Sobre 217 respuestas obtenidas, un 72 $\%$ de las personas que conocían el programa Tarjeta Blanca coincidieron en señalar que favoreció los valores trabajados durante el año (respeto, autonomía, motivación, igualdad, 
autoestima, salud y compañerismo). Para nosotros es un aspecto fundamental tener una opinión favorable de las familias, ya que consideramos de vital importancia su implicación y colaboración en todas las iniciativas que planteamos. También hay que señalar que el modelo de competición es aceptado de una manera excelente dentro de nuestro contexto, tal y como demuestra la encuesta $(85 \%$ de las familias considera que el modelo de competición favorece la formación integral y la transmisión de valores positivos). Durante la siguiente temporada 2011-2012, el proyecto se ha consolidado, ascendiendo muy significativamente el número de Tarjetas Blancas entregadas (308 conductas positivas premiadas para un total de 358 participantes). Hay que destacar que el número de jornadas celebradas en esta segunda temporada ha sido igual al de la primera (13).

Por otro lado, en el grupo de trabajo realizado con los entrenadores/as, estos expresaron también que sentían que potenciaba más aún su labor de transmisores de valores educativos a través de la práctica del baloncesto. El profesorado también mencionó que es un proyecto que funciona mejor en las primeras etapas, sobre todo con el alumnado de categoría benjamín y alevín, que con los jóvenes de categorías superiores. Se resaltó también la importancia de la implicación de las familias, siendo necesario que las conductas ensalzadas sean dadas a conocer a los padres y madres del alumnado premiado. Los resultados obtenidos nos han ayudado a mejorar y enriquecer este proyecto que consideramos puede sentar las bases para quien quiera trabajar en un futuro en esta misma línea. Como ejemplo de conductas que se premiaron en la última edición del torneo exponemos las siguientes. Estas frases, redactadas por los propios alumnos/as premiados, han sido extraídas literalmente de los tablones habilitados para tal efecto en la instalación:

- Fernando De Cea del equipo "Dragones": "Por ayudar a un compañero del otro equipo a levantarse" (es importante destacar que Fernando utiliza en su descripción la palabra compañero para referirse a un rival).

- Lucía Mandianes del equipo "Los Champiñons": "Por avisar al contrario en dos ocasiones de que no trate de sacar de debajo de la canasta porque va a rebotar el balón y va a perderlo".

- Carlos Castillo del equipo "Sek-Cadete": "Por ofrecerme a jugar con los chicos del otro equipo para el amistoso y mostrar una actitud ejemplar”.
- Álvaro Bernárdez del equipo "Lakers Quebrantahuesos": "Por reconocer que me habían hecho un tapón y no era falta, pese a que mis compañeros decían que sí”.

- Ignacio Hernández del equipo "Súper 8": "Por pararme cuando le di un codazo sin querer a un rival para ver si estaba bien", (y además le dio el balón para que sacara).

Por último, creemos importante seńalar que durante la jornada de Clausura del Torneo, se hizo entrega a algunos jugadores/as (bien de forma individual o a equipos completos) de un diploma honorífico por sus conductas deportivas, tanto por acumulación de comportamientos como por el significado de los mismos.

\section{Discusión}

Como proyecto innovador que planteamos, las referencias y experiencias previas en este ámbito son muy escasas. Destacamos la única referencia que hemos encontrado con respecto al uso de una tarjeta (en este caso de color "verde") en el ámbito deportivo y con fines de un reforzamiento de conductas positivas es el caso mencionado por Galeano (2001) con relación a la práctica deportiva en Finlandia. Sin embargo, este caso resulta mucho menos desarrollado, ya que no se explica el procedimiento de aplicación ni su contenido. Por otro lado, dentro de las diferentes estrategias que se han extrapolado al mundo deportivo desde la teoría del reforzamiento conductual (Skinner, 1985) y su ampliación a la teoría de los incentivos sociales (Bandura, 1987), el uso de "tarjetas" está muy arraigado en un gran número de deportes. No obstante, este recurso se ha orientado a penalizar conductas negativas guardando una noción de castigo. Este proyecto aporta un giro de $180^{\circ}$ respecto al uso de la "tarjeta", y convierte la Tarjeta Blanca en un elemento de promoción de valores sociales y personales excepcional a través de la competición.

Paralelamente a todo esto, consideramos que el programa ha mejorado nuestro conocimiento sobre las actitudes y comportamientos deportivos que pueden darse en la iniciación deportiva. Gracias a toda esa información, hemos podido elaborar un catálogo de buenos comportamientos y hábitos deportivos. En resumen, la experiencia y resultados obtenidos en estas dos primeras temporadas del proyecto han sido muy satisfactorios, y nos alientan a seguir trabajando con este programa.

\section{Referencias}

Bandura, A. (1987). Pensamiento y acción. Fundamentos sociales. Barcelona: Martínez Roca.

Carr, W. y Kemmis, S. (1988). Teoría crítica de la enseñanza. La investigación-acción en la formación el profesorado. Barcelona: Martínez Roca.

Durán, L.J. (2006). La actividad física y el deporte: Una oportunidad para transmitir valores. En CSD (ed.) Valores en movimiento. La actividad física y el deporte como medio de educación en valores, (45), 9-23. Madrid: Consejo Superior de Deportes.

Elliot, J. (1993). El cambio educativo desde la investigación-acción. Madrid: Morata.

Fundación Realmadrid. (2012). Por una Educación REAL: Valores y Deporte. Realmadrid, (39), 42-47. 
Galeano, E. (27 de abril de 2001) Los atletas químicos, El Mundo, 27-4-01. Jiménez, A.C. y Ortega, G. (2010). Evolución de un modelo de competición en una escuela de baloncesto, Wanceulen: Educación Física Digital, 7. Recuperado de http://www.wanceulen.com/ef-digital-numeros-anteriores/ ef-digital-n7-diciembre-2010.

Ortega, G. (2007) Aportaciones para la formación del profesorado en la iniciación al baloncesto: perspectiva profesional (Escuelas de baloncesto de la Fundación Real Madrid). En A.C. Jiménez, y G. Ortega (Eds.), Baloncesto en la Iniciación. (pp. 95-104). Sevilla: Wanceulen.

Ortega G., Sáenz-López, P., Durán, L.J., Giménez, F.J., Jiménez, P.J. y Jiménez, A.C. (2011). Programa de educación en valores a través de la enseñanza del deporte: un reto posible. En J. M. Román, M. A. Carbonero y J. D. Valdivieso (Eds.), Educación, Aprendizaje y Desarrollo en una Sociedad Multicultural. (pp.10.839-10.853). Madrid: Ediciones de la Asociación Nacional de Psicología y Educación.

Skinner, B.F. (1985). Aprendizaje y Comportamiento. Barcelona: Martínez Roca. 